NASA Contractor Report 4510

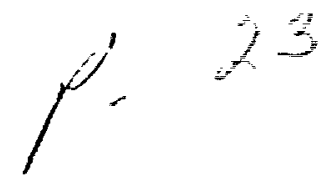

\title{
Inclusion of Transverse Shear \\ Deformation in the Exact \\ Buckling and Vibration Analysis of Composite Plate Assemblies
}

Melvin S. Anderson and David Kennedy

CONTRACT NAS1-18584 and

COOPERATIVE AGREEMENT NCCW-000002

MAY 1993

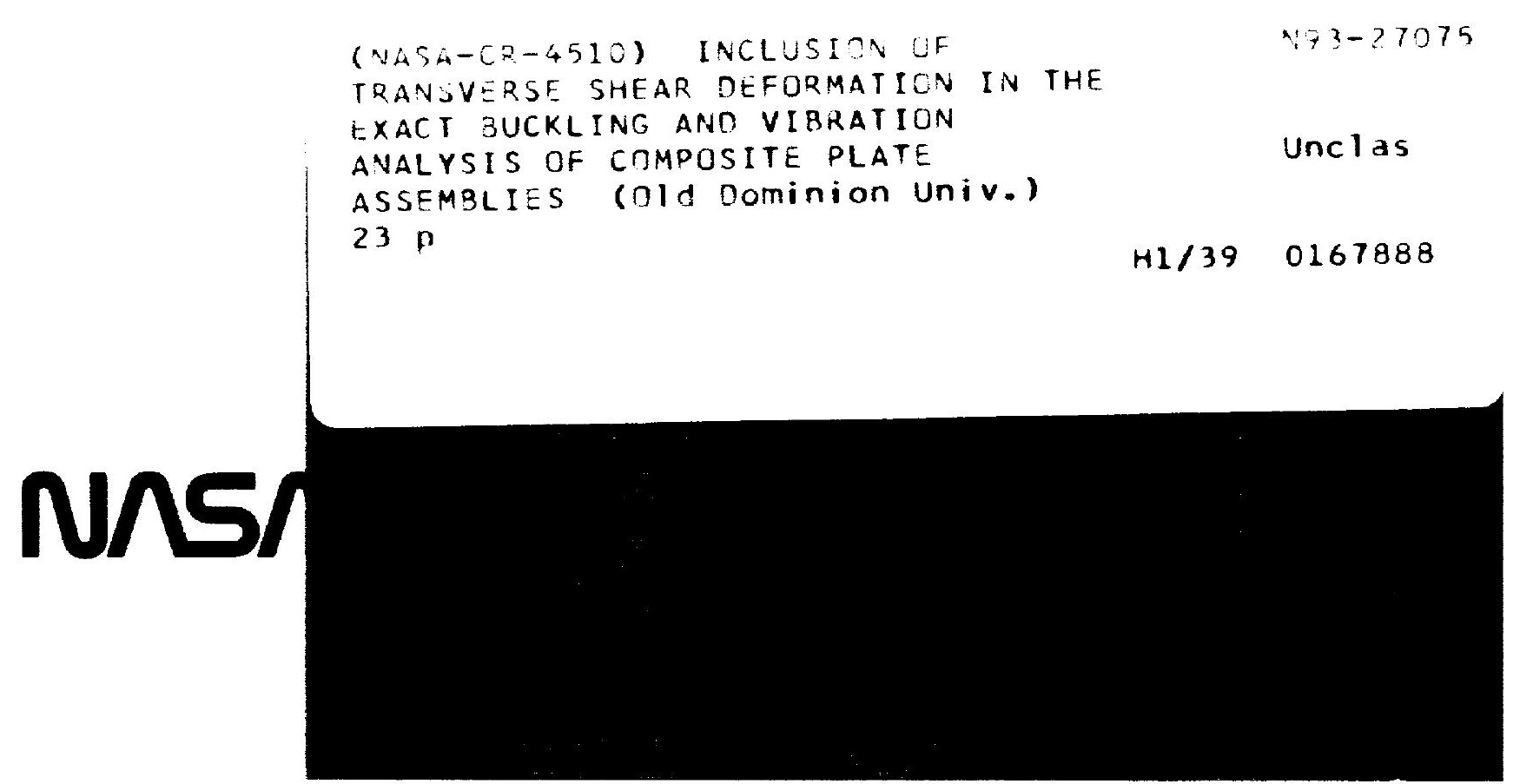




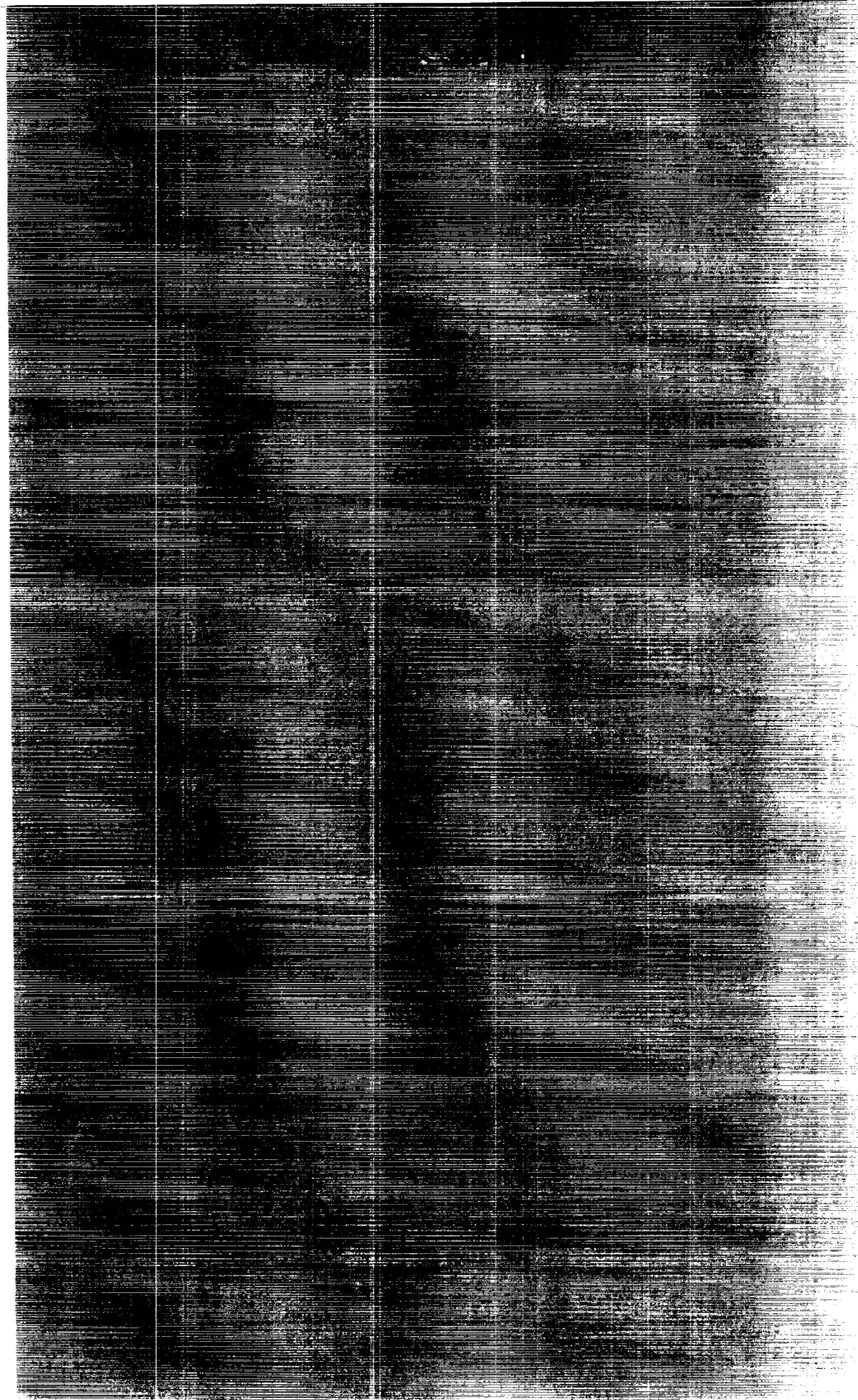


NASA Contractor Report 4510

\section{Inclusion of Transverse Shear \\ Deformation in the Exact \\ Buckling and Vibration Analysis of Composite Plate Assemblies}

Melvin S. Anderson

Old Dominion University Research Foundation

Norfolk, Virginia

David Kennedy

University of Wales College of Cardiff

Cardiff, United Kingdom

Prepared for

Langley Research Center

under Contract NAS1-18584 and

Cooperative Agreement NCCW-000002

\section{NASA}

National Aeronautics and

Space Administration

Office of Management

Scientific and Technical

Information Program

1993 

Inclusion of Transverse Shear Deformation in the Exact Buckling and Vibration Analysis of Composite Plate Assemblies

\author{
Melvin S. Anderson* \\ Old Dominion University Research Foundation \\ David Kennedy** \\ University of Wales College of Cardiff
}

\begin{abstract}
The problem considered is the development of the necessary plate stiffnesses for use in the general purpose program VICONOPT for buckling and vibration of composite plate assemblies. The required stiffnesses include the effects of transverse shear deformation and are for sinusoidal response along the plate length as required in VICONOPT. The method is based on the exact solution of the plate differential equations for a composite laminate having fully populated $\mathbf{A}, \mathbf{B}$, and $\mathbf{D}$ stiffness matrices which leads to an ordinary differential equation of tenth order.
\end{abstract}

\title{
Introduction
}

The VICONOPT program is described in reference 1 and is summarized in reference 2. The program performs buckling and vibration analysis of any prismatic assembly of composite plates and, optionally, the optimization of such plate assemblies. The assumptions made in the analysis are that the response in the longitudinal or $x$ direction is sinusoidal and that individual plates have stiffness properties that result from balanced symmetric laminates though asymmetric laminates are treated approximately. For orthotropic plate assemblies without shear loading, the sinusoidal response is exact for simply supported end conditions. For other cases, a series of sinusoidal modes is used with a Lagrangian multiplier technique to obtain results for quite general support conditions and loadings. The use of the term "exact" in the title refers to the fact that the analysis uses stiffness matrices that result from the exact solution of the uncoupled inplane and out-of-plane plate differential equations derived from classical plate theory. Because each of these equations are of fourth order, analytical

* Research Professor, NASA Langley Research Center, Hampton, VA 23681

** Lecturer / Senior Research Associate, School of Engineering, PO Box 925 ,
Cardiff CF2 1YF, United Kingdom 
expressions can be written for these stiffnesses. The introduction of transverse shear deformation increases the order of the out-of-plane differential equation to six so that a numerical approach is required to obtain its solution and the subsequent plate stiffnesses. Once the numerical approach is chosen, it can be developed in a general way to include the coupled case which is of tenth order when transverse shear is included and of eighth order otherwise. The coupled case allows the treatment of any laminate exactly with an arbitrary location of its reference surface.

The standard numerical approach for solution of the coupled plate equations with or without transverse shear is to write the plate equilibrium equations in terms of displacements, assuming a sinusoidal response in one coordinate direction to obtain a set of ordinary differential equations with independent variable the coordinate in the other direction. The characteristic roots are obtained by setting to zero the determinant of the coefficients of the displacement variables. This approach leads to increasingly long and complicated expressions that are impractical to implement for the most general cases. For plates with fully populated A, B, and D stiffness matrices, the number of terms is of the order ten thousand when transverse shear is included. The approach herein is to write the differential equations as a first order system with the displacements and the associated forces as unknowns as was done by Cohen in reference 3 . The resulting matrix has typically two or three terms in each matrix element for the most general case. These equations can be arranged so that the characteristic root of the differential equation system appears only on the diagonal, thus a standard linear eigenvalue solver can be used. The eigenvectors then give the relationships between all the forces and displacements.

Because exact expressions are used for stiffnesses, there is no need to subdivide a plate into segments as is done with conventional finite element methods to obtain accuracy. Nodes are required only where different plates are joined, usually at an angle with one another. The assumptions of plate theory are such that continuity of rotation at the junction of two or more plates requires that the shear angle in the plane of the edge forming the junction must be zero unless all plates are coplanar. A key step in the analysis is to use the shear angle as the additional unknown over classical plate theory, as was done by Cohen in reference 3 . After calculation of the plate stiffness matrix, the rows and columns corresponding to the shear angle are simply removed so that the remaining unknowns are the same as classical plate theory. Thus the part of the computer code involving assembly of the global stiffness matrix from individual plate stiffnesses is 
unchanged from that used for classical plate theory. Only additional routines to calculate the plate stiffnesses including transverse shear need to be developed.

\section{Theory}

\section{Governing Plate Equations}

It is assumed that the plate has fully populated $\mathbf{A}, \mathbf{B}$, and D stiffness matrices with a reference surface arbitrarily located in the $x-y$ plane as shown in figure 1. The plate is loaded by uniform inplane stress resultants $\mathrm{N}_{\mathrm{x}}, \mathrm{N}_{\mathrm{y}}$, and $\mathrm{N}_{\mathrm{x} y}$ that act in a centroidal plane shown in figure $\mathrm{l}(\mathrm{a})$. The centroidal plane is located at a distance $z_{c}$ from the reference surface and passes through the centroid determined from beam theory considering the plate to be wide beam whose length is in the $x$ direction. Figure $1(b)$ shows the additional inplane forces $n_{x}, n_{y}, n_{x y}$, moments $m_{x}, m_{y}, m_{x y}$, and transverse shearing forces $q_{x}$, $q_{y}$ that occur during buckling or the amplitudes of these forces and moments when vibration at a frequency of $\omega$ is considered. The deflections $u, v$, and $w$ of the reference surface are in the $x, y$, and $z$ directions respectively. For vibration or buckling, deflection in all three directions is resisted by Winkler elastic foundations of stiffness $\mathrm{K}_{\mathrm{x}}, \mathrm{K}_{\mathrm{y}}$, and $\mathrm{K}_{\mathrm{z}}$. The equations of equilibrium are

$\left.\mathrm{n}_{\mathrm{x}, \mathrm{x}}+\mathrm{n}_{\mathrm{xy}, \mathrm{y}}-\mathrm{N}_{\mathrm{x}} \mathrm{u}_{0}\right), \mathrm{xx}-\mathrm{K}_{\mathrm{x}} \mathrm{u}+4 \pi^{2} \omega^{2}\left(\mathrm{~m}_{0} \mathrm{u}-\mathrm{m}_{1} \psi_{\mathrm{x}}\right)=0$

$n_{x y, x}+n_{y}, y-N_{x} v_{0}, x x-K_{y} v+4 \pi^{2} \omega^{2}\left(m_{0} v-m_{1} \psi_{y}\right)=0$

$q_{x, x}+q_{y, y}-K_{z} w-N_{x} w, x x-N_{y} w, y y-2 N_{x y} w, x y+4 \pi^{2} \omega^{2} m_{0} w=0$

$m_{x y, x}+m_{y, y}-q_{y}-N_{x} z_{c} v_{0}, x x+4 \pi^{2} \omega^{2}\left(m_{1} v-m_{2} \psi_{y}\right)=0$

$\left.m_{x, x}+m_{x y}, y-q_{x}-N_{x} z_{C} u_{(}\right), x x+4 \pi^{2} \omega^{2}\left(m_{1} u-m_{2} \psi_{x}\right)=0$

where a comma indicates differentiation with respect to the variables that follow, $u_{0}$ and $v_{0}$ are inplane deflections at the centroidal plane, $m_{j}$ is the jth moment of mass about the reference surface and $\psi_{x}$ and $\psi_{y}$ are rotations of the normals to the reference surface about the $y$ and $x$ faces, respectively. These equations are generalized from those of reference 4 to allow for the applied load $N_{x}$ to act at the centroidal surface which may be different from the reference surface. As in reference 4 , it is assumed that the only inplane force to affect the inplane equilibrium equations is $N_{X}$. The inplane displacements in the centroidal plane, $u_{0}$ and $v_{0}$, are given by 


$$
\begin{aligned}
& \mathrm{u}_{0}=\mathrm{u}-\mathrm{z}_{\mathrm{c}} \psi_{\mathrm{x}} \\
& \mathrm{v}_{0}=\mathrm{v}-\mathrm{z}_{\mathrm{c}} \psi_{\mathrm{y}}
\end{aligned}
$$

\section{Stiffness Matrix}

The objective of the analysis is to derive a stiffness matrix that relates the force quantities along the edges $y= \pm \frac{b}{2}$ to the displacements along the same edges. (Because of the sinusoidal variation in the $x$ direction, the stiffness matrix will involve the amplitude of these quantities).

The desired displacement variables are

$$
\mathbf{d}=\left[\begin{array}{cc}
i & u \\
v \\
w \\
\psi_{y} \\
i & \gamma_{x}
\end{array}\right]
$$

where $\gamma_{\mathrm{x}}=\mathrm{w}, \mathrm{x}-\psi_{\mathrm{x}}$ has been introduced as a fundamental displacement variable rather than the rotation $\psi_{X}$ in order to preserve continuity of rotations along nodal lines corresponding to plate junctions.

The corresponding force variables are

$$
f=\left[\begin{array}{cc}
i & n_{x} y \\
& n_{y} \\
Q_{y} \\
m_{y} \\
i & m_{x y}
\end{array}\right]
$$

The imaginary number $\mathrm{i}$ has been introduced so that the phase shift between the various quantities that occurs for orthotropic plates without shear loading results in real plate stiffnesses for such plates. The transverse shearing edge force in the $z$ direction, denoted as $Q_{y}$, must be normal to the reference surface of the undeflected plate. This is accomplished by replacing $q_{y}$ with the Kirchhoff value 


$$
q_{y}=Q_{y}-m_{x y, x}+N_{y} w, y+N_{x y} w, x
$$

Note that the $m_{x y, x}$ term which is found in the Kirchhoff shear term of classical plate theory is also present in the transverse shear case when $\gamma_{X}$ is used as a fundamental displacement variable. (This fact can be shown from the principle of virtual work).

The problem is changed to an ordinary differential equation in $y$ by assuming a sinusoidal variation in the $x$ direction. If the variables in equations (3) and (4) are considered to be functions of $y$, the displacements and forces throughout the plate are given by

$$
\mathbf{Z}(x, y)=\exp \left(\frac{i \pi x}{\lambda}\right) z(y)
$$

where

$$
z=\left[\begin{array}{l}
d \\
f
\end{array}\right]
$$

and $\lambda$ is the half-wavelength of the response in the $x$ direction. The next step is to express all unknowns in terms of $\boldsymbol{z}$. The stress resultant strain relationships given in terms of the $\mathbf{A}, \mathbf{B}$, and $\mathbf{D}$ stiffness matrices are partially inverted to give needed quantities in terms of the fundamental variables or terms derivable from fundamental variables without any $y$

$$
\begin{aligned}
& {\left[\begin{array}{c}
\mathrm{n}_{\mathrm{x}} \\
\varepsilon_{\mathrm{y}} \\
\varepsilon_{\mathrm{x} y} \\
\mathrm{~m}_{\mathrm{x}} \\
\kappa_{\mathrm{y}} \\
\kappa_{\mathrm{x} y}
\end{array}\right]=\left[\begin{array}{cccccccccccc}
\mathrm{h}_{1} & 1 & \mathrm{~h}_{1} & 2 & \mathrm{~h}_{1} & 3 & \mathrm{~h}_{1} & 4 & \mathrm{~h}_{1} & 5 & \mathrm{~h}_{1} & 6 \\
-\mathrm{h}_{1} & 2 & h_{2} & 2 & h_{2} & 3 & h_{2} & 4 & h_{2} & 5 & h_{2} & 6 \\
-h_{1} & 3 & h_{2} & 3 & h_{3} & 3 & h_{3} & 4 & h_{3} & 5 & h_{3} & 6 \\
h_{1} & 4 & -h_{2} & 4 & -h_{3} & 4 & h_{4} & 4 & h_{4} & 5 & h_{4} & 6 \\
-h_{1} & 5 & h_{2} & 5 & h_{3} & 5 & -h_{4} & 5 & h_{5} & 5 & h_{5} & 6 \\
-h_{1} & 6 & h_{2} & 6 & h_{3} & 6 & -h_{4} & 6 & h_{5} & 6 & h_{6} & 6
\end{array}\right]\left[\begin{array}{c}
\varepsilon_{x} \\
n_{y} \\
n_{x y} \\
\kappa_{x} \\
m_{y} \\
m_{x y}
\end{array}\right]} \\
& {\left[\begin{array}{c}
q_{x} \\
\gamma_{y}
\end{array}\right]=\left[\begin{array}{ccccc}
h_{7} & 7 & h_{7} & 8 \\
-h_{7} & 8 & h_{8} & 8
\end{array}\right]\left[\begin{array}{l}
\gamma_{x} \\
q_{y}
\end{array}\right]}
\end{aligned}
$$


where

$$
\begin{aligned}
& \varepsilon_{\mathrm{x}}=\mathrm{u}, \mathrm{x} \\
& \kappa_{\mathrm{x}}=-\psi_{\mathrm{x}}, \mathrm{x} \\
& \psi_{\mathrm{x}}=\mathrm{w}, \mathrm{x}-\gamma_{\mathrm{x}}
\end{aligned}
$$

The constants $h_{i j}$ appearing in the first of equations (7) can be calculated from the standard $\mathbf{A}, \mathbf{B}$, and $\mathbf{D}$ stiffness matrices of laminate theory. The shear stiffness terms in the last two equations are determined from reference 5 .

It is also necessary to write equation (5) without the appearance of any $y$ derivatives. The result is

$$
q_{y}=\frac{\left(Q_{y}-m_{x y, x}+N_{y}\left(\psi_{y}-\gamma_{x} h_{78}\right)+N_{x y}\right)}{1-N_{y} h_{8} 8}
$$

The five strain displacement equations are

$$
\begin{aligned}
& \varepsilon_{x y}=u, y+v, x \\
& \varepsilon_{y}=v, y \\
& \gamma_{y}=w, y-\psi_{y} \\
& \kappa_{y}=-\psi_{y}, y \\
& \kappa_{x y}=\gamma_{x}, y-\gamma_{y}, x-2 \psi_{y}, x
\end{aligned}
$$

Using equations (7) and (8), the strain-displacement and equilibrium equations can be written in terms of the elements of $\boldsymbol{z}$ as follows

$$
\mathbf{z}^{\prime}=\mathbf{P} \mathbf{z}
$$

where a prime denotes differentiation with respect to $y$. The elements of $\mathbf{z}$ are assumed to be given by

$$
z_{j}=c_{j} \exp \left(\frac{i \beta y}{b}\right)
$$

where $\beta$ is a characteristic root of the differential equation with as many values as the order of the differential equation system. Substituting equation (11) into equation (10) results in 


$$
(\mathbf{R}-\beta \mathbf{I}) \mathbf{c}=0
$$

where $I$ is the identity matrix and $\mathbf{c}$ is the vector formed from the $c_{j}$ of are the (11). Thus, the characteristic roots of the differential equation multiplying or dividing appropriate not symmetric but can be made real by of $\mathbf{R}$ are given in Appendix deformation cases and it can for both the classical and transverse shear easy to implement in a computer seen that they are not complicated and are associated eigenvector computer program. For each eigenvalue, the matrix with columns as the eigenv to the $c_{j}$ of equation (12). If $\mathbf{C}$ is a be associated with displacements antors, the upper half, denoted as a, will associated with forces as follows

$$
\mathbf{C}=\left[\begin{array}{l}
\mathbf{a} \\
\mathbf{b}
\end{array}\right]
$$

Denoting quantities evaluated at $y=-b / 2$ by superscript 1 and quantities evaluated at $y=b / 2$ by superscript 2 , the amplitudes of the

$$
\begin{aligned}
& \mathrm{d}_{\mathrm{j}}^{\mathrm{j}}=\sum_{\mathrm{k}=1}^{N} \mathrm{ajk}_{\mathrm{j}} \mathrm{r}_{\mathrm{k}} \exp \left(\frac{-\mathrm{i} \beta_{\mathrm{k}}}{2}\right) \\
& \mathrm{d}_{\mathrm{j}}^{2}=\sum_{\mathrm{k}=1}^{N} \mathrm{a}_{j k} \mathrm{r}_{\mathrm{k}} \exp \left(\frac{\mathrm{i} \beta_{\mathrm{k}}}{2}\right) \\
& \mathrm{f}_{\mathrm{j}}^{\mathrm{l}}=\sum_{\mathrm{k}=1}^{N} \mathrm{~b}_{\mathrm{jk}} \mathrm{r}_{\mathrm{k}} \exp \left(\frac{-\mathrm{i} \beta_{k}}{2}\right) \\
& \mathrm{f}_{\mathrm{j}}^{2}=\sum_{\mathrm{k}=1}^{N} \mathrm{~b}_{\mathrm{jk}} \mathrm{r}_{\mathrm{k}} \exp \left(\frac{\mathrm{i} \beta_{\mathrm{k}}}{2}\right)
\end{aligned}
$$


where $r_{k}$ are constants to be determined from the edge values and $\mathrm{N}$ is the order of the differential equation. In matrix form, equations (14) are written as

$$
\begin{aligned}
& {\left[\begin{array}{l}
d^{1} \\
d^{2}
\end{array}\right]=E r} \\
& {\left[\begin{array}{l}
f^{1} \\
f^{2}
\end{array}\right]=F r}
\end{aligned}
$$

Eliminating $\mathbf{r}$ from equations (15) and (16) gives

$$
\left[\begin{array}{l}
f^{1} \\
f^{2}
\end{array}\right]=K\left[\begin{array}{l}
d^{1} \\
d^{2}
\end{array}\right]
$$

where $\mathbf{K}$ is the desired stiffness matrix given by

$$
\mathbf{K}=\mathbf{F} \mathbf{E}^{-1}
$$

The rows and columns of $\mathbf{K}$ corresponding to $\gamma_{\mathrm{X}}$ are deleted to obtain a stiffness matrix of the same size and involving the same unknowns as for the classical case. An alternate procedure that yields a lower bound solution is to reduce the stiffness matrix to the same final unknowns by setting $m_{x y}$ to zero which is an option available in the program. (For the case of two plates that are coplanar, the substructuring capability of the program may be used with five degrees of freedom at each edge to join the two plates before the extra degree of freedom is removed). As for the classical case, $\mathbf{K}$ is real and symmetric for orthotropic plates without shear loading and is Hermitian otherwise. The resulting stiffness matrix can be used without further alteration in the original coding for the classical case.

Obtaining accurate numerical results for $\mathbf{K}$ from equation (18) can be a problem for certain ranges of parameters. The methods used to overcome these problems is discussed in Appendix B.

\section{Eigenvalues for Clamped Edges}

The analysis procedure used in VICONOPT described in reference 1 is an iterative procedure that requires the plate stiffnesses to be evaluated at a series of trial values of the eigenvalue (load factor for buckling or frequency for vibration and not to be confused with the eigenvalues of the 
$\mathbf{R}$ matrix) that converge to the desired result. For each trial value, the analysis requires not only the plate stiffness but also the number of
eigenvalues exceeded for each individual clamped. (See referer each individual plate assuming its edges were finding the buckling or vibration complete discussion of the algorithm for obtain the eigenvalues of the indion eigenvalue). It would be quite difficult to most general case by applying standard analysis techniques edges for the approach used herein was developed in analysis techniques. The to be subdivided into smaller eloped in reference 4 and requires the plate guarantee that none of the subdivided with a width small enough to exceeded. Using this divided subdivided plate clamped edge eigenvalues are doubled to return to the original width the procture that is repeatedly allows the number of eigenvalues width, the procedure of reference 4 determined.

To implement this procedure, a suitable number of subdivisions is istermined as follows. Appendix $A$ shows that every term of the $\mathbf{R}$ matrix is proportional to the plate width $b$, and thus the eigenvalues of $\mathbf{R}$ are proportional to $\mathrm{b}$. Noting that an eigenvalue equal to $\pi$ corresponds to buckling or vibration with simply supported edges, successively halving b until all the real eigenvalues of $\mathbf{R}$ are less than $\pi$ gives the width of a plate for which no eigenvalues are exceeded if the edges are simply supported. and consequently none are exceeded if the edges are clamped.

\section{Results}

Results have been obtained for single sandwich plates with isotropic face sheets and agree exactly with published results in the literature for
various boundary conditions.

The accuracy of the transverse shear theory for use in eigenvalue problems is indicated by comparison with the results of reference 6 where three dimensional elasticity results were obtained for comparison with various shear deformation theories. The exact elasticity results are compared in table 1 for the vibration of a composite simply supported composed of ten equal thickness layers with the ply angles alternating length direction and circumferential waves one axial half-wave in the 1 to 10 . The first column in the table is having harmonics varying from $\mathrm{n}$, the second column is the error in the present results relative to number elasticity solution and the third in the present results relative to the 
the frequency obtained neglecting transverse shear deformation. It can be seen that for a ratio of wall thickness $h$ to cylinder radius $r$ of .05 which is representative of a relatively thick shell, the effect of transverse shear is still small until $\mathrm{n}$ becomes large. The error for the present results is quite small for the entire range. For $h / r=.3$ which is well beyond proportions usually encountered, the effect of transverse shear is appreciable but the present results have errors of only about two per cent. These results are in agreement with the conclusion of reference 6 that first order shear deformation theory is adequate to predict global response such as vibration or buckling. The present results were obtained by subdividing the circular arcs into small segments and linking them together by substructuring techniques retaining all five degrees of freedom at an edge.

Table 1 Accuracy of shear deformation theory for cylinder vibration.

\begin{tabular}{|c|c|c|c|c|}
\hline \multirow[b]{2}{*}{$\mathrm{n}$} & \multicolumn{2}{|c|}{$\mathrm{h} / \mathrm{r}=.05$} & \multicolumn{2}{|c|}{$\mathrm{h} / \mathrm{r}=.3$} \\
\hline & Error, \% & $\frac{\omega}{\omega_{c}}$ & Error, \% & $\frac{\omega}{\omega_{\mathrm{cl}}}$ \\
\hline 1 & -.39 & .996 & .95 & .818 \\
\hline 2 & -.06 & .996 & 1.56 & .716 \\
\hline 3 & -.17 & .989 & 1.96 & .660 \\
\hline 4 & .30 & .977 & 2.29 & .589 \\
\hline 5 & .51 & .955 & 2.39 & .520 \\
\hline 6 & .70 & .932 & 2.31 & .463 \\
\hline 7 & .89 & .909 & 2.20 & .426 \\
\hline 8 & 1.05 & .884 & 1.98 & .449 \\
\hline 9 & 1.23 & .859 & 1.72 & .487 \\
\hline 10 & 1.39 & .834 & 1.46 & .521 \\
\hline
\end{tabular}

The effect of shear deformation is of particular importance when low density cores are used to form a structural sandwich. The following example illustrates the increased efficiency and the importance of transverse shear deformation for such structures.

The problem considered is a flat panel with blade (web) stiffeners designed to carry an axial load of $5000 \mathrm{lb} / \mathrm{in}$. acting as a simply supported wide column of 30 inch length. The composite material used has stiffness properties $\mathrm{E}_{1}=19 . \times 10^{6}, \mathrm{E}_{2}=1.89 \times 10^{6}, \mathrm{E}_{12}=.93 \times 10^{6}, \mathrm{E}_{13}=\mathrm{E}_{12}$. $\mathrm{E}_{23}=.5 \mathrm{E}_{12}$, all with units psi. and Poisson ratio $v=.31$. The subscript 1 refers to the fiber direction, the subscript 2 refers to transverse to the fiber in the laminate plane, and subscript 3 refers to normal to the 
laminate plane The density is $.0571 \mathrm{lb} / \mathrm{cu}$ in. The sandwich core material is available in a range of densities which result in a varying shear stiffness. A typical variation of the shear stiffness of a foam core suitable for sandwich construction is shown in figure 2. The foam core was assumed to have a constant Young's modulus of $1000 \mathrm{psi}$ at all densities with Poisson ratio of zero. Using these properties, optimized designs were determined for solid composite and sandwich construction assuming both classical and transverse shear deformation theory. Typical cross-sections resulting from these analyses are shown in figure 3 where the relative size of one bay of the wide column panel is shown along with the actual panel dimensions. Only the design based on transverse shear theory is shown for the solid composite as it differs little from that based on classical theory. The composite laminates considered were $\left[ \pm 45^{\circ} / 0^{\circ}\right] \mathrm{S}$ for each plate with a central foam core added for the sandwich configurations. The thickness of the various layers are given using the subscripts 45 or 0 for the composite material and a $c$ to indicate the total thickness of the foam core for the sandwich configurations. For sandwich construction, the efficiency is such that unrealistically high strains result if the design is unconstrained so allowable strain limits were imposed of .006 along a fiber, .004 normal to a fiber and .005 for shear strain. These limits led the optimizer to reduce the thickness of the $\pm 45^{\circ}$ plies to zero so they were omitted in the sandwich designs.

The mass of the various designs as a function of core density is shown in figure 4. Designs based on classical theory show a monotonic increase of mass with core density since the shear modulus of the core does not affect the result. Accounting for transverse shear results in a minimum mass at about ten $\mathrm{lb} / \mathrm{cu} \mathrm{ft}$ core density. However there is a range of core densities that can achieve nearly the minimum mass. The upper line showing the mass of the solid composite panel is about 1.6 times the minimum mass achievable with sandwich construction. For the higher core densities and the solid composite, there is very little difference in the mass achievable using classical or transverse shear theory. However even for these cases, a panel that just meets the design buckling load using classical theory would have a buckling load 5 to 10 per cent below the design value if analyzed using transverse shear theory.

The sandwich cross-sections shown in figure 3 were obtained for a core density of $6.9 \mathrm{lb} / \mathrm{cu} \mathrm{ft}$ so they are in the range where transverse shear effects are significant. In this case, using classical theory to design the panel (the middle configuration of figure 3 ) results in a configuration that 
would buckle at only $65 \%$ of the buckling load design requirement if analyzed using transverse shear theory.

\section{Concluding Remarks}

A numerical approach to obtain exact plate stiffnesses including the effects of transverse shear has been presented. The generality of the method allows inclusion of fully populated A, B, and D stiffness matrices. These stiffnesses have been incorporated in the VICONOPT program for analysis and optimization of composite panels. Before the incorporation of this new capability, VICONOPT was limited to plates analyzed with classical theory having a zero $B$ matrix so the addition of the new capability is an improvement for cases when classical theory is used as well as the transverse shear case.

Two key items in the approach are:

(1) expressing the necessary equations as a first order system involving the displacement and force quantities desired in the final stiffness relation.

(2) using the shear strain rather than a rotation for the extra degree of freedom introduced by transverse shear.

The first item allowed the characteristic roots of the differential equation to be obtained from a relatively simple matrix. This is in contrast to previous conventional approaches for this type of problem which could lead to matrices whose elements consist of over ten thousand terms for the tenth order system associated with the coupled case including transverse shear. The second item allows the plate stiffness matrix to be reduced to the same size as that for classical plate theory because continuity of rotation requires the shear strain to be zero a the junction of two plates that are not coplanar. A junction having all, lates coplanar is treated by creating substructures with all degrees of freedom present and eliminating the extra degrees of freedom before assembly in the final stiffness matrix. Thus no additional coding is required to assemble the global stiffness matrix and to solve the resulting eigenvalue problem.

Results of the analysis applied to the vibration of a composite cylinder show that the shear deformation theory used gives results with little error compared to the elasticity solution. The analysis applied to a typical design problem showed that sandwich construction can give significant mass savings compared to solid composite construction. The analysis makes possible the determination of optimum core density for sandwich 
construction and indicates the need to account for shear deformations especially when the lower mass core materials are used.

Acknowledgment- This work was supported by the National Aeronautics and Space Administration under contract NAS1-18584-05 and Cooperative Agreement NCCW-000002. The work of the second author was also supported by British Aerospace plc. The authors would especially like to acknowledge the input of Gerald Flanagan, Grumman Aircraft Systems Division, who made available his inhouse research on shear deformation theory applied to composite panels which was a valuable guide in developing the present analysis. Also they wish to thank Professor F. W. Williams, University of Wales College of Cardiff for suggesting the problem and his consultation in the analysis development.

\section{Appendix A \\ Matrix for Characteristic Roots}

The eigenvalues of $\mathbf{R}$ of equation (12) are the characteristic roots of the differential equations governing plate behavior. The nonzero elements of the 10 by 10 matrix $\mathbf{R}$ are

$$
\begin{aligned}
& r_{11}^{\#}=r_{66}^{\#}=-\alpha h_{13} \\
& r_{12}^{\#}=r_{76}^{\#}=-\alpha \\
& r_{13}^{\#}=-r_{86}^{\#}=-\frac{\alpha}{b^{r}} 15=-\frac{\alpha}{b^{r}} 106=-\frac{\alpha^{2}}{b} h_{34} \\
& r_{16}^{\#}=-b_{3} 3 \\
& r_{17}^{\#}=r_{26}^{\#}=-b_{2} 3 \\
& r_{19}^{\#}=-r_{46}^{\#}=-b_{3} 5 \\
& r_{110}=r_{56}=-b_{36} \\
& \mathrm{r}_{21}^{\#}=\mathrm{r}_{67}^{\#}=-\alpha \mathrm{h}_{12} \\
& \mathrm{r}_{23}^{\#}=-\mathrm{r}_{87}^{\#}=-\frac{\alpha}{\mathrm{b}^{\mathrm{r}} 25}=-\frac{\alpha}{\mathrm{b}^{\mathrm{r}} 107}=-\frac{\alpha^{2}}{\mathrm{~b}} \mathrm{~h}_{24} \quad \mathrm{r}_{27}^{\#}=-\mathrm{bh}_{22} \\
& \text { r } 29=-r_{4}^{\#}=-b_{2} 5 \\
& r_{33}=r_{88}=\frac{\alpha N_{x y} h_{88}}{S} \\
& \mathrm{r}_{210}=\mathrm{r}_{57}=-\mathrm{bh}_{2} 6 \\
& \mathrm{r} 34=\mathrm{r} 98=\frac{\mathrm{b}}{\mathrm{S}} \\
& r_{35}=-r_{1} 08=-\frac{b h_{78}}{S} \\
& \mathrm{r}_{4}^{\#}{ }_{1}=-\mathrm{r}_{69}^{\#}=\alpha \mathrm{h}_{15} \\
& r_{4}^{\#}=r_{89}^{\#}=-\frac{\alpha}{b^{r}} 45=\frac{\alpha}{b^{r}} 109=-\frac{\alpha^{2}}{b} h_{45} \\
& \mathrm{r}_{4} 9=\mathrm{bh}_{5} 5 \\
& \mathrm{r}_{310}=-\mathrm{r} 58=-\frac{\alpha}{\mathrm{b}^{\mathrm{r}} 38}=-\frac{\alpha \mathrm{h}_{88}}{\mathrm{~S}} \\
& \mathrm{r}_{410}=-\mathrm{r}_{59}=\mathrm{bh}_{56}
\end{aligned}
$$




$$
\begin{aligned}
& \mathrm{r}_{51}=\mathrm{r}_{610}=-\alpha \mathrm{h}_{16} \\
& r_{53}=-r_{810}=\frac{\alpha^{2}}{b}\left(h_{46}+\frac{N_{x y} h_{88}}{S}\right) \\
& \text { r54 }=- \text { r9 } 10=\alpha+\frac{\alpha}{\mathrm{S}} \\
& r_{55}=r_{1} 010=-\alpha\left(h_{46}+\frac{h_{7} 8}{S}\right) \\
& \mathrm{r}_{510}=-\mathrm{bh}_{66}-\frac{\alpha^{2} \mathrm{~h}_{88}}{\mathrm{bS}} \\
& \mathrm{r}_{6}^{\#} 1=-\mathrm{B}_{\mathrm{X}}+\frac{\alpha^{2} \mathrm{~h}_{11}}{\mathrm{~b}} \\
& r_{63}^{\#}=-r_{81}^{\#}=-\frac{\alpha}{b} r_{65}=-\frac{\alpha}{b} r_{1} 01=-\frac{\alpha^{3} h_{14}}{b^{2}}+\alpha X_{1} \\
& \mathrm{r} \frac{\# 2}{72}=-\mathrm{B}_{\mathrm{y}} \\
& r \#_{4}=-r \#_{2}=b X_{1} \\
& \mathrm{r}_{83}=\mathrm{B}_{\mathrm{Z}}+\alpha^{2} \mathrm{X}_{2}+\frac{\alpha^{2} \mathrm{~N}_{\mathrm{Xy}}^{2} \mathrm{~h}_{88}}{\mathrm{bS}} \\
& \mathrm{r}_{84}=\mathrm{r}_{93}=\frac{\alpha \mathrm{N}_{\mathrm{X}} \mathrm{S}}{\mathrm{S}} \\
& r_{85}=-r_{103}=-\alpha b X_{2}-\frac{\alpha N_{x y} h_{78}}{S} \\
& r_{94}=\frac{\alpha^{2}}{b} h_{44}+b^{2} X_{2}+\frac{b N_{y}}{S} \\
& \text { r95 }=-r_{104}=-\frac{b_{y} h_{78}}{S} \\
& r_{105}=-b^{2} X_{2}+b h_{77}-\frac{b N_{x y} h_{78}^{2}}{S}
\end{aligned}
$$

where

$$
\begin{aligned}
& \alpha=\frac{\pi b}{\lambda} \\
& S=1-N_{y} h_{88} \\
& X_{1}=\pi^{2}\left(4 m_{1} \omega^{2}+\frac{N_{x} z_{c}}{\lambda^{2}}\right) \\
& \left.X_{2}=\pi^{2}+\frac{m_{2} \omega^{2}}{b}+\frac{N_{x} z_{c}^{2}}{b \lambda^{2}}\right)-\frac{\alpha^{2}}{b^{3} h_{44}} \\
& B_{\mu}=\pi^{2} b\left(4 m_{0} \omega^{2}+\frac{N_{x}}{\lambda^{2}}\right)-b K_{\mu}
\end{aligned}
$$

where $\mu$ can be $x, y$ or $z$.

Equations for the classical case can be obtained by setting the transverse shear strains, $\gamma_{\mathrm{x}}$ and $\gamma_{\mathrm{y}}$, equal to zero and noting that $\psi_{\mathrm{x}}=w, \mathrm{x}$. The partially inverted stress strain relations corresponding to equations (7) are slightly different in that $m_{x y}$ and $\kappa_{x y}$ are interchanged. Only the first four equilibrium equations from equations (1) are used (the fifth is satisfied by 
incorporation into the final form of the third). Following the same steps as for the transverse shear case, the matrix whose eigenvalues are the characteristic roots is of order eight. Elements with a superscript \# previously given for the transverse shear case apply also to the classical case if 1 is subtracted from any index greater than 4 . The remaining elements not given above are

$$
\begin{array}{ll}
\mathrm{r}_{14}=-\mathrm{r}_{85}=-2 \alpha \mathrm{h}_{36} & \mathrm{r}_{24}=-\mathrm{r}_{86}=-2 \alpha \mathrm{h}_{26} \\
\mathrm{r}_{34}=\mathrm{r}_{87}=\mathrm{b} & \mathrm{r}_{44}=\mathrm{r}_{88}=2 \alpha \mathrm{h}_{56} \\
\mathrm{r}_{54}=-\mathrm{r}_{81}=-\frac{2 \alpha^{2}}{\mathrm{~b}} \mathrm{~h}_{16} & \mathrm{r}_{73}=\mathrm{B}_{\mathrm{z}}+\alpha^{2} \mathrm{X}_{2} \\
\mathrm{r}_{74}=\mathrm{r}_{83}=\alpha \mathrm{N}_{\mathrm{xy}}-\frac{2 \alpha^{3}}{\mathrm{~b}^{2}} \mathrm{~h}_{46} & \mathrm{r}_{84}=\alpha \frac{\mathrm{h}_{44}-4 \mathrm{~h}_{66}}{\mathrm{~b}}+\mathrm{b}^{2} \mathrm{X}_{2}+\mathrm{bN}_{\mathrm{y}}
\end{array}
$$

\section{Numerical Solution of Equations}

There are several possibilities of numerical problems arising in determining $\mathbf{K}$ given in equation (18) as

$$
\mathbf{K}=\mathbf{F} \mathbf{E}^{-1}
$$

First the eigenvectors of the matrix $\mathbf{R}$ which form the elements of $\mathbf{E}$ and $\mathbf{F}$ must be determined accurately for extreme proportions and a wide range of scaling. With accurate values of the elements of $\mathbf{E}$ and $\mathbf{F}$, the solution procedure is as follows. Solve the system of equations

$$
\mathbf{E}^{*} \mathbf{x}=\mathbf{F}^{*}
$$

The solution is

$$
\mathbf{x}=\left(\mathbf{E}^{*}\right)^{-1} \mathbf{F}^{*}=\mathbf{K}^{*}
$$

where superscript $*$ indicates Hermitian transpose of the matrix. The solution is accomplished by Gaussian elimination with pivoting on the largest current diagonal except as will be discussed subsequently for transverse shear cases. A typical element in row $k$ in the matrix equation (B3) has a multiplying factor of $\exp \left(\frac{+i \beta_{k}}{2}\right)$ where $\beta_{k}$ is the $k^{\text {th }}$ characteristic root. If the imaginary part of $\beta_{k}$ is too large, numeric overflow will occur. To prevent this, a real number is subtracted from the argument of each 
exponential in the $\mathrm{k}^{\text {th }}$ row such that overflow will not occur for the particular computer being used. This has the effect of multiplying the $k^{\text {th }}$ equation by a constant which does not change the solution.

An additional problem occurs for transverse shear cases where the transverse shear effects are small. One pair of characteristic roots becomes very large and the remainder approach the values for the classical case. Finite element formulations have had serious problems for this case and much effort has been spent to avoid errors from too stiff elements associated with locking that can occur with routine application of theory. This problem is handled for the present case by placing the terms involving these large roots in the last two rows of equation B2 and not including these rows in the logic for pivoting on the largest diagonal. This approach has proved to be quite successful, stiffnesses and buckling loads having been accurately calculated for aluminum plates with widththickness ratios as high as one thousand.

In some cases, particularly for unloaded isotropic plates, the characteristic roots $\beta$ from equation 12 occur in repeated pairs and the above procedure breaks down. For this case small perturbations in the positive and negative directions are made to all three foundation stiffnesses $\left(\mathrm{K}_{\mathrm{x}}, \mathrm{K}_{\mathrm{y}}, \mathrm{K}_{\mathrm{z}}\right.$ in equation 1) in order to separate the repeated roots. The stiffness matrix is obtained by interpolation between the two stiffness matrices resulting from the perturbed foundation stiffnesses.

\section{References}

1. Williams, F. W., Kennedy, D., and Anderson, M. S., "Analysis features of VICONOPT, an exact buckling and vibration program for prismatic assemblies of anisotropic plates," Proceedings of the $31 \mathrm{st}$ AIAA/ASME/ ASCE/AHS/ASC Structures, Structural Dynamics and Materials Conference, Long Beach, CA, pp. 920-929, 1990. AIAA paper 90-0970

2 Williams, F. W., Kennedy, D., Butler, R., and Anderson, M. S., "VICONOPT: Program for exact vibration and buckling analysis or design of prismatic plate assemblies," AIAA Journal, Vol. 29, No. 11, 1991, pp. 1927-1928. 
3. Cohen, G. A. , "FASOR- A second generation shell of revolution code," Computers \& Structures Vol. 10, 1979, pp. 301-309.

4. Wittrick, W. H., and Williams, F. W., "Buckling and vibration of anisotropic plate assemblies under combined loadings." International Journal Mechanical Sciences, Vol. 16, 1974, pp. 209-239.

5. Cohen, G. A., "Transverse shear stiffness of laminated anisotropic shells," Computer Methods in Applied Mechanics and Engineering, Vol. 13, 1978, pp. 205-220.

6. Noor, A. K., Burton, W. S., and Peters, J. M., "Predictor-Corrector procedures for stress and free vibration analyses of multilayered composite plates and shells," Computer Methods in Applied Mechanics and Engineering, Vol. 82, 1990, pp. 341-363. 


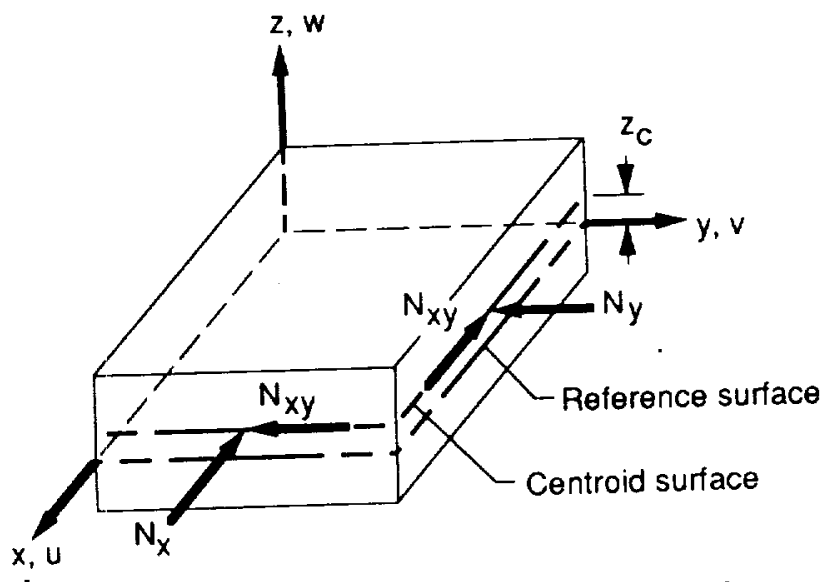

(a)Prebuckling inplane loads

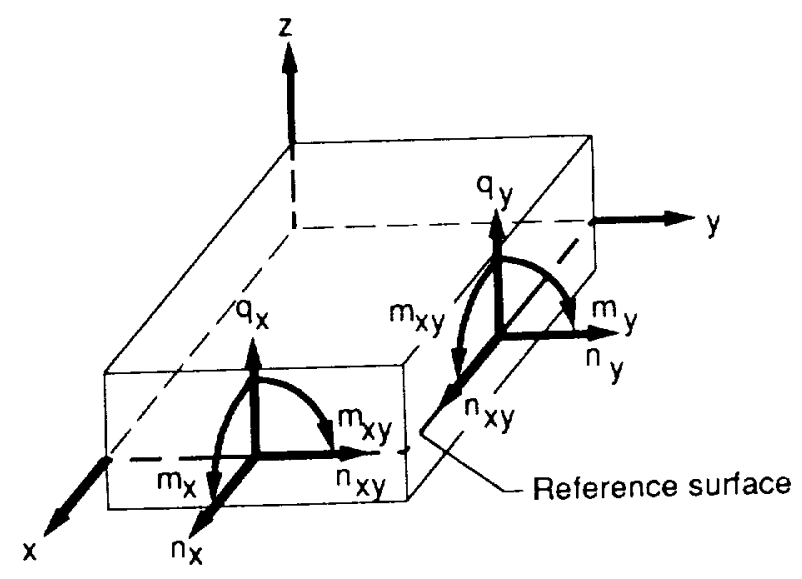

(b) Buckling forces and moments

Figure 1. Positive direction of forces and moments per unit width acting on a plate element.

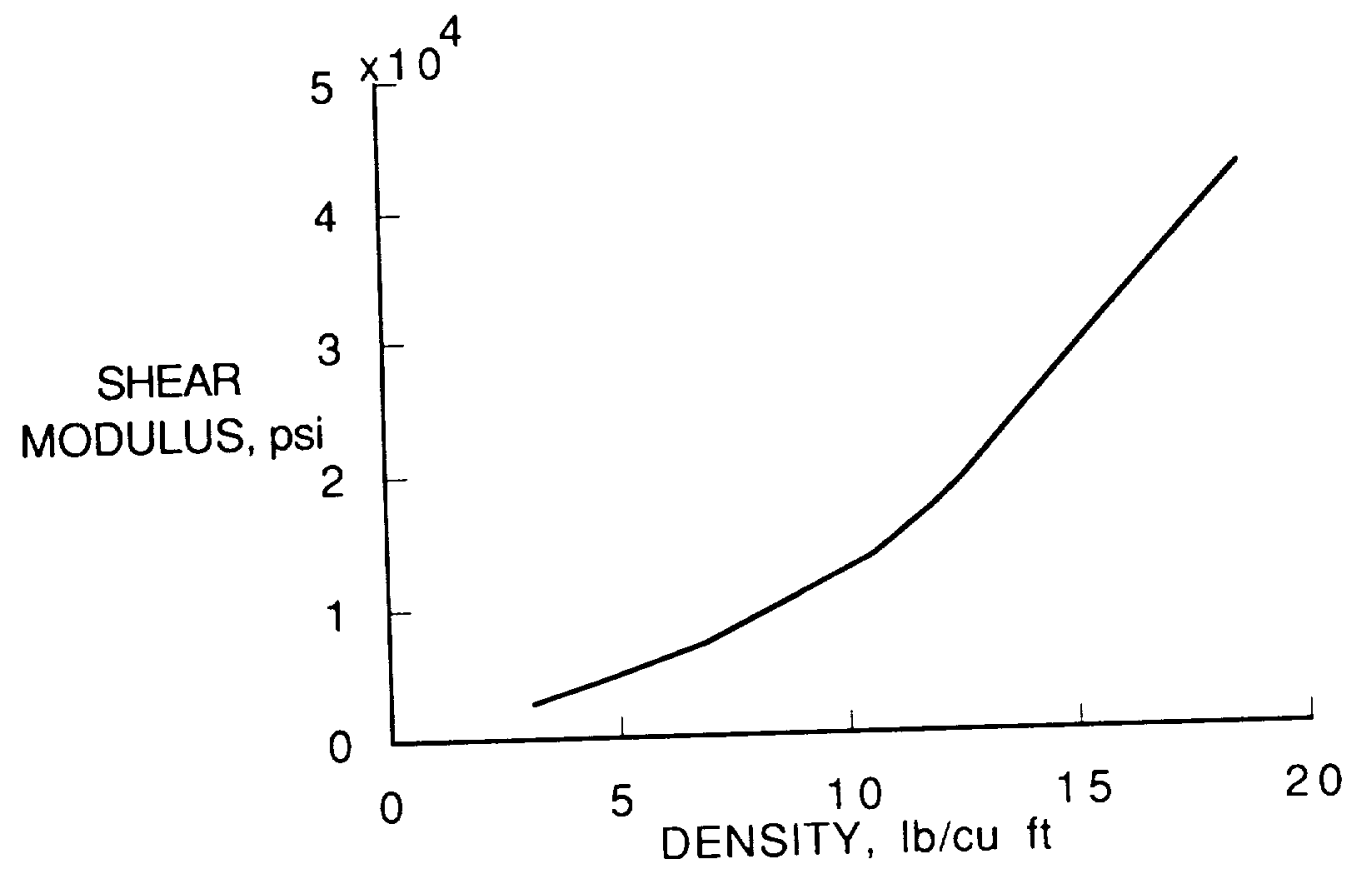

Figure 2. Shear modulus variation of foam core material with density. 


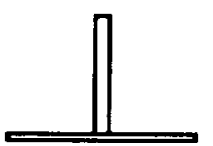

Solid

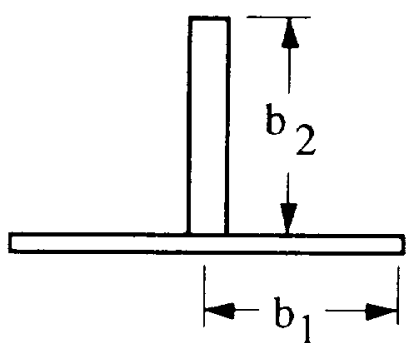

Sandwich

Classical Theory

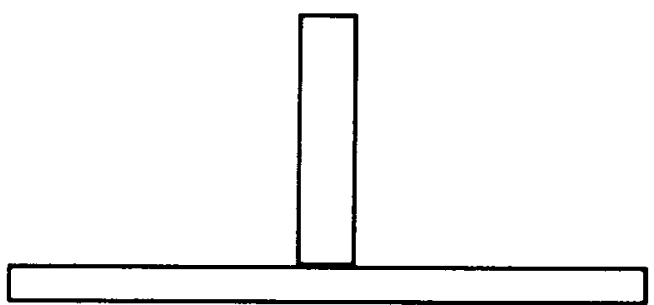

Sandwich

Transverse Shear Theory

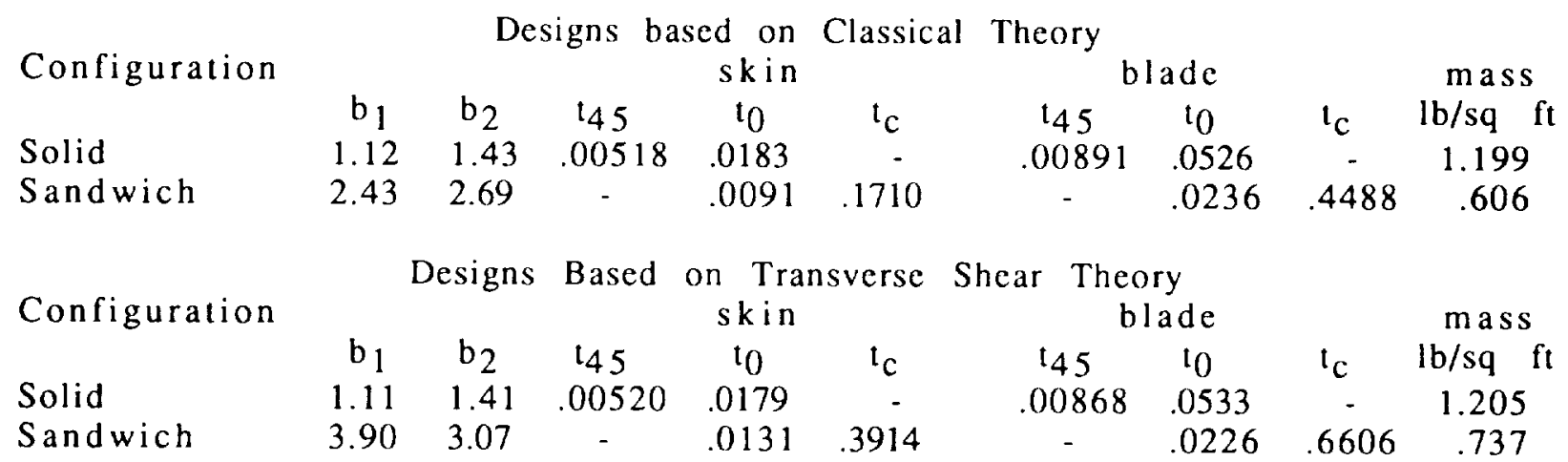

Figure 3. Geometry of solid composite and sandwich configurations designed to carry axial loading of $5000 \mathrm{lb} / \mathrm{in}$. Sandwich core density is 6.9 $\mathrm{lb} / \mathrm{cu} \mathrm{ft}$ corresponding to a shear modulus of $7100 \mathrm{psi}$. Panel length is 30 inches. All dimensions are in inches 


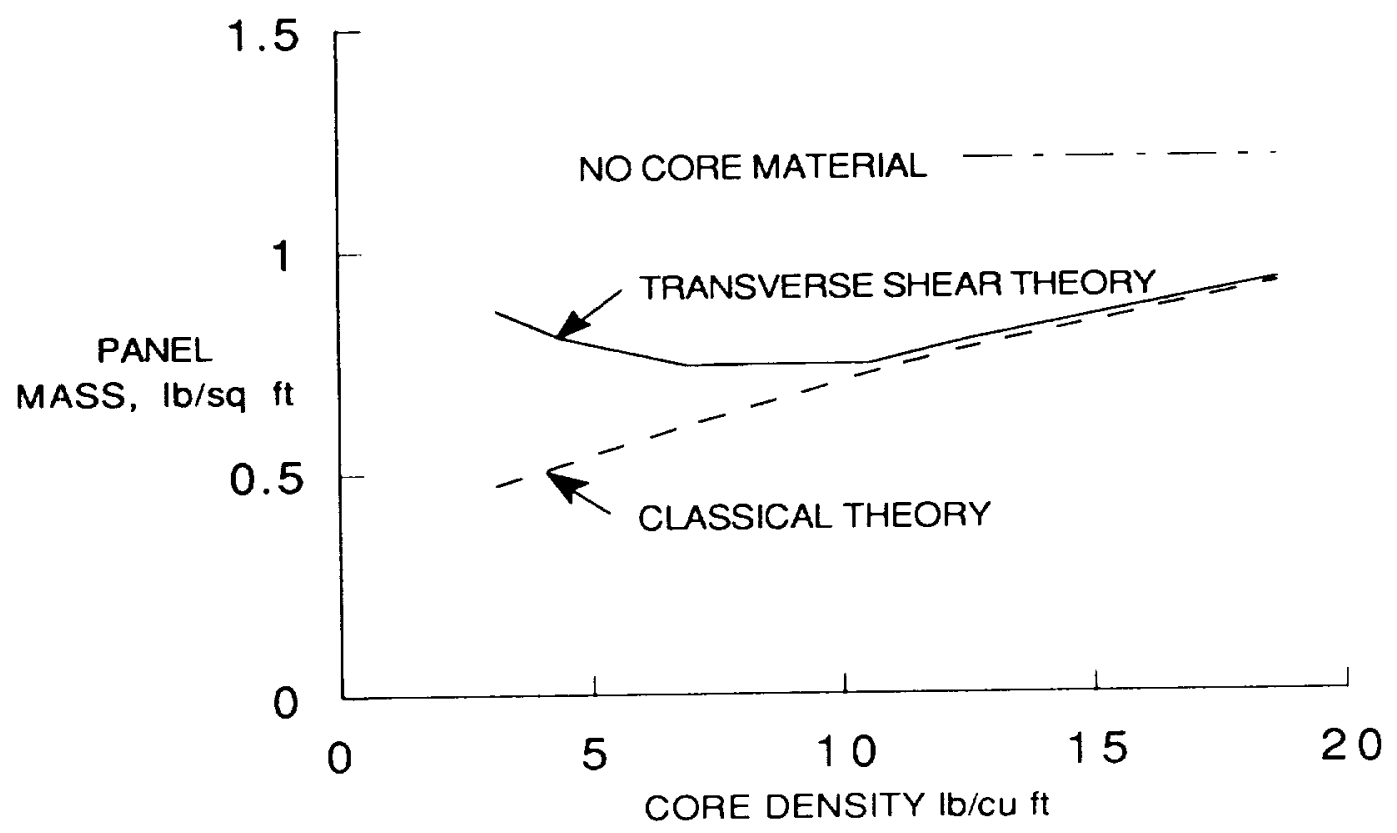

Figure 4. Minimum panel mass as a function of core density. 



\begin{tabular}{|c|c|c|c|c|}
\hline \multicolumn{3}{|c|}{ REPORT DOCUMENTATION PAGE } & \multicolumn{2}{|r|}{$\begin{array}{l}\text { Form Approved } \\
\text { OMB No. 0704-0188 }\end{array}$} \\
\hline \multicolumn{5}{|c|}{ 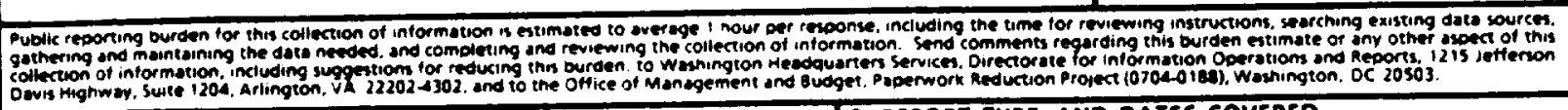 } \\
\hline 1. AGENCY USE ONLY (Leave blank) & $\begin{array}{l}\text { and to the OHice or Mondegement and } \\
\text { 2. REPORT DATE } \\
\text { MaV } 1993\end{array}$ & \multicolumn{3}{|c|}{$\begin{array}{l}\text { 3. REPORT TYPE AND DATES COVERED } \\
\text { Contractor ReDort }\end{array}$} \\
\hline \multicolumn{3}{|c|}{$\begin{array}{l}\text { 4. TITLE AMD SUDTITLE } \\
\text { Inclusion of Transverse Shear Deformation in the } \\
\text { Exact Buckling and Vibration Analys is of Composite } \\
\text { Plate Assemblies }\end{array}$} & \multicolumn{2}{|c|}{$\begin{array}{l}\text { C NAS } 1-18584 \\
\text { TA } 05\end{array}$} \\
\hline \multicolumn{3}{|c|}{ 6. AUTHOR(S) } & \multicolumn{2}{|c|}{ WU 505-63-50-07 } \\
\hline \multicolumn{3}{|c|}{$\begin{array}{l}\text { 0ld Dominion University Research Foundation } \\
\text { Norfolk, VA } 23508 \text { and } \\
\text { University of Hales College of Cardiff } \\
\text { P. 0. Box } 925 \text {, Cardiff CF2 IYF, United Kingdom }\end{array}$} & \multicolumn{2}{|c|}{$\begin{array}{l}\text { 8. PERFORMING ORGANIZATION } \\
\text { REPORT NUMBER }\end{array}$} \\
\hline \multicolumn{3}{|c|}{$\begin{array}{l}\text { 9. SPOMSORING/MONITOAIMG AGEMCY NAME(S) AND ADDAESS(ES) } \\
\text { National Aeronaut ics and Space Administration } \\
\text { Langley Research Center } \\
\text { Hampton, VA } 23681-0001\end{array}$} & \multicolumn{2}{|c|}{$\begin{array}{l}\text { 10. SPONSORING/MONITOAING } \\
\text { AGENCY REPORT NUMBER } \\
\text { NASA CR-4510 }\end{array}$} \\
\hline \multicolumn{5}{|c|}{$\begin{array}{l}\text { 11. SUPPLEMETARY NOTES } \\
\text { Langley Technical Monitor: James H. Starnes, Jr. } \\
\text { Melvin S. Anderson: Old Dominion University Research Foundation, Norfolk, VA } \\
\text { David Kennedy: Univers ity of Wales College of Cardiff, United Kingdom }\end{array}$} \\
\hline \multicolumn{3}{|c|}{$\begin{array}{l}\text { 12a. DASTRIBUTION/AVAILABLITY STATEMENT } \\
\text { Unclassified - Unl imited } \\
\text { Subject Category - } 39\end{array}$} & \multicolumn{2}{|c|}{ 12b. DISTRIBUTION CODE } \\
\hline \multicolumn{5}{|c|}{$\begin{array}{l}\text { 13. ADstract (Maximum 200 words) } \\
\text { The problem considered is the development of the necessary plate stiffnesses for use in the } \\
\text { general purpose program VICONOPT for buckling and vibration of composite plate } \\
\text { assemblies. The required stiffnesses include the effects of transverse shear deformation and } \\
\text { are for sinusoidal response along the plate length as required in VICONOPT. The method is } \\
\text { based on the exact solution of the plate differential equations for a composite laminate having } \\
\text { fully populated A, B, and D stiffness matrices which leads to an ordinary differential equation } \\
\text { of tenth order. }\end{array}$} \\
\hline \multirow{2}{*}{\multicolumn{4}{|c|}{$\begin{array}{l}\text { 14. SUDECT TERMS } \\
\text { composite plates, buckling, transverse shear deformation }\end{array}$}} & $\begin{array}{l}\text { 15. MUMBER OF PAGES } \\
24\end{array}$ \\
\hline & & & & $\begin{array}{l}\text { 16. PAICE COOE } \\
\text { A03 }\end{array}$ \\
\hline \multirow{2}{*}{$\begin{array}{l}\text { 17. SECUAITY QASSIFICATION } \\
\text { Of REPOAT } \\
\text { Unclassified }\end{array}$} & $\begin{array}{l}\text { D. SECURITY CLASSIFICATION } \\
\text { OF THIS PAGE }\end{array}$ & \multirow{2}{*}{\multicolumn{2}{|c|}{$\begin{array}{l}\text { 19. SECURITY CQASSIFICATION } \\
\text { Of AESTRACT } \\
\text { Unclass ified }\end{array}$}} & 20. LIMITATION OF AESTRACT \\
\hline & Unclassified & & & \\
\hline
\end{tabular}


National Aeronautics and

Space Administration

Code JTT

Washington, D.C.

20546-0001

Official Business

BULK RATE

POSTAGE \& FEES PAID

NASA

Permit No G.27

Penalty for Private Use, $\$ 300$ 


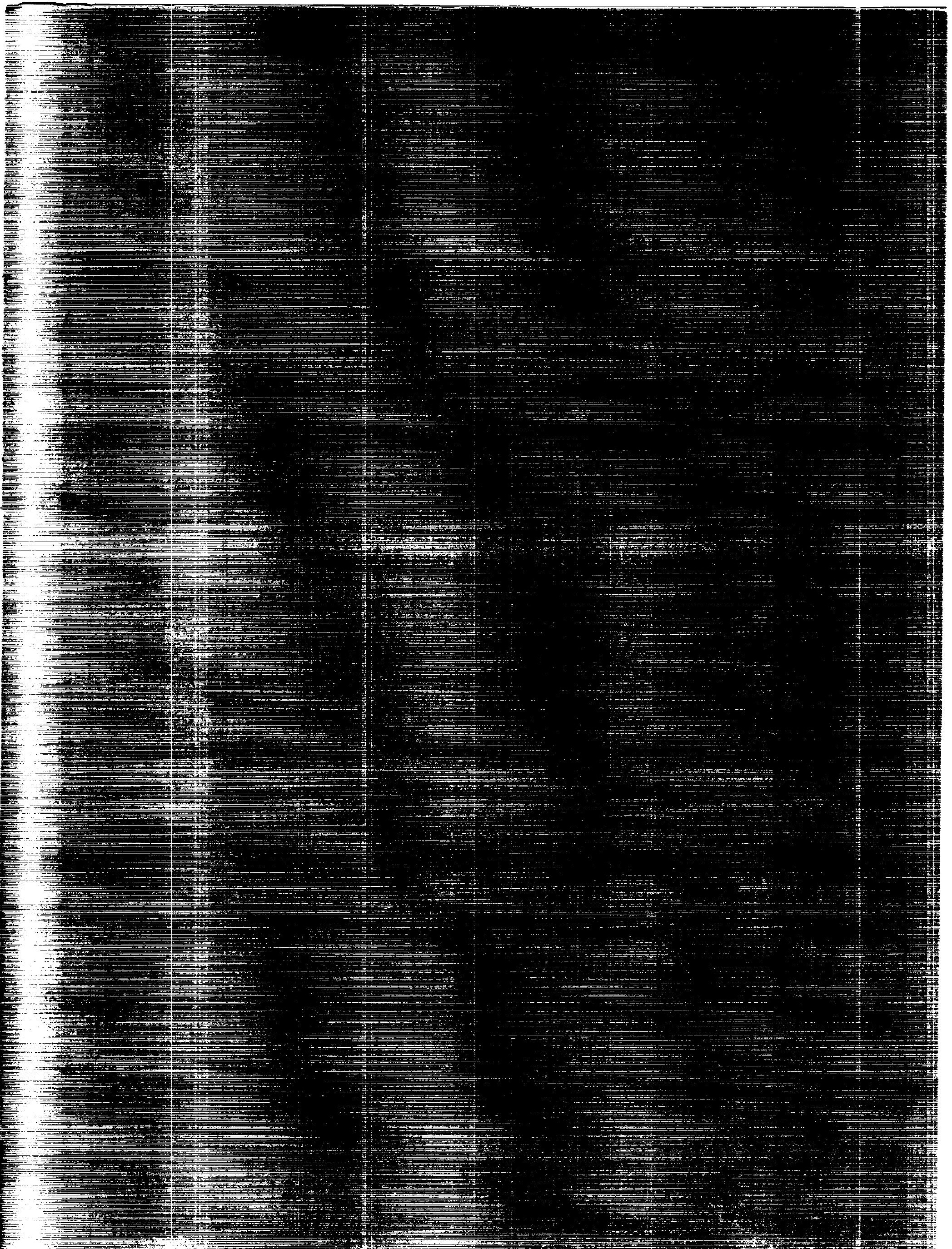

